\title{
Comparison of the preventive effects of pretreatment of lidocaine with a tourniquet and a premixed injection of lidocaine on propofol-LCT/MCT injection pain
}

\author{
Hyo Jin Byon, Kil Woo Lee, Hee Yong Shim, Jang Ho Song, Jong Kwon Jung, Young Deog Cha, and \\ Doo Ik Lee
}

Department of Anesthesiology and Pain Medicine, Inha University College of Medicine, Incheon, Korea

Background: Lidocaine has been used widely to prevent propofol injection pain. Various methods of administration exist, such as lidocaine premixed with propofol or lidocaine pretreatment using a tourniquet, but it is unclear which method of lidocaine administration is more effective for the prevention of injection pain of propofol LCT/MCT. The purpose of this study was to compare pretreatment of lidocaine with a tourniquet and a premixed injection of lidocaine to prevent injection pain of propofol-LCT/MCT.

Methods: Patients were randomly allocated into the pretreatment group $(\mathrm{n}=117)$ or the premixed group $(\mathrm{n}=117)$. The pretreatment group was pretreated with $2 \mathrm{ml}$ of lidocaine $2 \%$, held with a tourniquet, before propofol-LCT/MCT injection. The premixed group was injected with a premixed solution of propofol-LCT/MCT and $2 \mathrm{ml}$ of lidocaine $2 \%$. To evaluate the incidence and severity of pain, spontaneous verbal expressions of pain, movement of hand, frowning, and moaning were recorded, and the patients were asked to recall their pain with the visual analogue score (VAS) 30 minutes after awakening from anesthesia.

Results: Overall, injection pain occurred in $13.7 \%$ of the pretreatment group and $15.4 \%$ of the premixed group, without any statistical difference $(\mathrm{P}=0.71)$. There was no difference in spontaneous verbal expressions of pain, movement of hand, frowning, and moaning between the two groups. The pain intensity (VAS) also showed no difference between the two groups $(\mathrm{P}=0.49)$.

Conclusions: Pretreatment of lidocaine with a tourniquet showed no more benefit to prevent injection pain of propofol LCT/MCT compared to a premixed injection with lidocaine. (Korean J Anesthesiol 2014; 66: 95-98)

Key Words: Emulsions, Injections, Lidocaine, Pain, Propofol, Tourniquets.

Received: May 13, 2013. Revised: July 2, 2013. Accepted: August 8, 2013.

Corresponding author: Jang Ho Song, M.D., Department of Anesthesiology and Pain Medicine, Inha University College of Medicine, 7-206, 3-ga, Sinheung-dong, Jung-gu, Incheon 400-711, Korea. Tel: 82-32-890-3937, Fax: 82-32-890-3968, E-mail: jhs@inha.ac.kr

(c) This is an open-access article distributed under the terms of the Creative Commons Attribution Non-Commercial License (http:// creativecommons.org/licenses/by-nc/3.0/), which permits unrestricted non-commercial use, distribution, and reproduction in any medium, provided the original work is properly cited. 


\section{Introduction}

Propofol is a widely used intravenous anesthesia induction agent well known for its safety and efficacy such as rapid onset and short duration. Nevertheless, intravenous injection of propofol induces injection pain in 60 to $90 \%$ of patients [1-4]. This leads to the patient's discomfort, even discontent with the anesthesia. There have been many studies trying to reduce injection pain $[5,6]$. Lidocaine has been reported for its preventive effect of injection pain during propofol administration [6]. Reports show that pretreatment with lidocaine with a tourniquet has a better effect than a premixed injection with propofol-LCT [2] but not consistently. Furthermore, propofol-LCT/MCT was introduced as a new chemical agent with less injection pain than propofol-LCT $[7,8]$, and lidocaine is effective for reducing injection pain of this agent [9]. However, no comparative studies have been done on the preventive effect of injection pain between a premixed injection and pretreatment of lidocaine during propofol-LCT/MCT administration in the same clinical setting

The purpose of this study was to determine which would reduce injection pain more during propofol-LCT/MCT administration, a premixed injection of lidocaine or pretreatment of lidocaine with a tourniquet.

\section{Materials and Methods}

After approval by the Institutional Review Board, this study included 234 patients from 20 to 65 years old, who were American Society of Anesthesiologists physical status I or II, injected with propofol for general anesthesia, and had given their written consent. The exclusion criteria were as follows: ischemic heart diseases, neurologic problems, pregnancy, breast-feeding women, administration of analgesics within 24 hours before surgery, allergic to propofol or its contents (soy-bean oil, MCT, glycerol, egg lecithin, and sodium oleate), and allergic to lidocaine.

The patients were divided into either a pretreatment group or premixed group using a randomization table generated by a computer program. All medicines were prepared and labeled by the institutional pharmacy, double blinding both the administrator and patients. The medicines were propofol-LCT/MCT (1\% Fresofol ${ }^{\circledR}$ Fresenius kabi, Graz, Austria [200 mg/20 ml]) and lidocaine HCL (Lidocaine ${ }^{\circledR}$ HCL 2\% Huons, Jecheon, Korea [400 $\mathrm{mg} / 20 \mathrm{ml}]$ ), and they were kept at room temperature.

Intravenous accesses were made on all patients on the day before or the very day of the surgery with an $18 \mathrm{G}$ intravenous catheter, and isotonic saline was infused by a maintenance rate. No premedications were given such as analgesics and sedatives.

When the patient arrived at the surgery room, standard monitoring for general anesthesia was applied. A tourniquet (Baumanometer ${ }^{\circledR}$, W.A.Baum Co. Inc, Copiague. NY, USA) was applied on the forearm at a pressure of $60 \mathrm{mmHg}$. The pretreatment group was injected with a $40 \mathrm{mg}$ solution of $2 \%$ lidocaine at the distal most part of the intravenous line, flushed with $3 \mathrm{ml}$ of normal saline. One minute later, propofol $2 \mathrm{mg} / \mathrm{kg}$ with $2 \mathrm{ml}$ of normal saline solution was injected. On the other hand, the premixed group was pretreated with a tourniquet with $2 \mathrm{ml}$ of normal saline, then flushed with $3 \mathrm{ml}$ of normal saline. Then one minute later, propofol $2 \mathrm{mg} / \mathrm{kg}$ with a $40 \mathrm{mg}$ solution of $2 \%$ lidocaine was injected. All of the agents were injected at the same rate of $1 \mathrm{ml} / \mathrm{sec}$, as the drug infusion rate may affect the incidence of propofol injection pain.

Sevoflurane with $100 \% \mathrm{O}_{2}$ was administered and manual ventilation was applied by an assisted or controlled mode. After administration of vecuronium $0.12 \mathrm{mg} / \mathrm{kg}$, the patient was intubated endotracheally. Sevoflurane in $50 \% \mathrm{O}_{2}$ with air was administered for maintenance of anesthesia.

The patients were not directly questioned for their discomfort or pain during anesthesia induction. Instead, the aspect of pain was observed. The aspects of pain in all patients were evaluated by the same investigator. Major pain criteria included verbal expressions of pain and movement of hand with a look of withdrawing from pain. Minor pain criteria included moaning and frowning [10]. After surgery, the patients were placed in the recovery room. Thirty minutes after awakening from anesthesia, they were asked to recall propofol injection pain during anesthesia induction, and the intensity of pain was evaluated with visual analogue score (VAS). Injection pain was defined when there was more than one major criteria or more than one minor criteria and a VAS > $1[10]$.

\section{Statistical analysis}

The appropriate sample size was estimated based on the assumption that we were interested in an incidence reduction of propofol-LCT/MCT injection pain by $15 \%$ with the premixed group having an incidence of $23 \%$ for propofol-LCT/MCT injection pain in a previous study $[7,11]$. This yielded a sample size of 105 subjects per group with $\alpha=0.05, \beta=0.8$, using Fisher exact test with the G-power program. Taking general patient loss into consideration, 117 patients were included in each group with 234 patients in total.

The incidence of injection pain and the patients' gender were compared using $\chi^{2}$ or Fischer's exact test between the two groups. The patients' demographic data (age, height and weight), blood pressure, heart rate, oxygen saturation and VAS were compared using the student's t-test or Mann-Whitney-Wilcoxon test. 


\section{Results}

All 234 adult patients were included, without any patient excluded for side effects. The two groups showed no statistical difference in demographic data (Table 1). Furthermore, there were no statistical differences in both major criteria and minor criteria defining the injection pain, nor in the severity of pain (VAS) when the patients were asked to recall their pain 30 minutes after awakening from anesthesia. Among the 34 patients showing injection pain, only 15 patients recalled their pain (premix group $7 / 18$, pretreatment group $8 / 16$ ). The two groups showed no statistical difference in overall incidence of pain (Table 2), and there was no significant difference in complication like hypotension or bradycardia.

\section{Discussion}

We conducted this study to compare pretreatment with a tourniquet and premixing of lidocaine to prevent injection pain while administering propofol-LCT/MCT. There was no statistical difference in the incidence and severity of propofol-LCT/ MCT injection pain between the pretreatment group and the premixing group. Some studies also reported pretreatment of lidocaine for one minute by applying a tourniquet at an adequate pressure is enough to prevent injection pain of propofol-LCT/ MCT $[6,12,13]$. Nevertheless, injecting a premixed solution of propofol-LCT/MCT and lidocaine does not require more time or an additional procedure. Thus, injecting this premixed solution is more advisable for a clinical setting, considering its simi-

Table 1. Demographic Data of Subjects

\begin{tabular}{lcc}
\hline & Group pretreat $(\mathrm{n}=117)$ & Group premix $(\mathrm{n}=117)$ \\
\hline Age $(\mathrm{yr})$ & $43.0[34.0-52.0]$ & $42.0[27.8-52.0]$ \\
Male $:$ Female & $60: 57$ & $65: 52$ \\
Height $(\mathrm{cm})$ & $163.0[157.0-171.0]$ & $165.0[159.3-171.8]$ \\
Weight $(\mathrm{kg})$ & $64.0[57.0-73.5]$ & $65.0[57.0-74.0]$ \\
\hline
\end{tabular}

Values expressed as median [interquartile range] or number.

Table 2. Pain Score

\begin{tabular}{lccc}
\hline & $\begin{array}{c}\text { Group pretreat } \\
(\mathrm{n}=117)\end{array}$ & $\begin{array}{c}\text { Group premix } \\
(\mathrm{n}=117)\end{array}$ & P value \\
\hline Verbal expression & $12(10.3 \%)$ & $13(11.1 \%)$ & 0.83 \\
Movement of hand & $6(5.1 \%)$ & $8(6.8 \%)$ & 0.58 \\
Frowning & $11(9.4 \%)$ & $6(5.1 \%)$ & 0.21 \\
Moaning & $7(6.0 \%)$ & $3(2.6 \%)$ & 0.20 \\
Propofol injection pain & $16(13.7 \%)$ & $18(15.4 \%)$ & 0.71 \\
Severity of pain (VAS)* & $0[0-3]$ & $2.5[0-3.25]$ & 0.49 \\
\hline
\end{tabular}

Values expressed as number (\%) of total patients of each group or median [interquartile range]. *VAS (Visual Analog Scale) was analyzed only in patients showing propofol injection pain. lar preventive effect compared with pretreatment of lidocaine using a tourniquet.

According to previous studies, propofol injection pain may vary depending on gender, age, intravenous injection vein site, and propofol concentration [1]. These factors may affect the incidence of propofol injection pain in the present study. We tried to reduce these potential sources of bias by injecting the same concentration of propofol-LCT/MCT via a venous catheter at the same site (dorsal hand vein). This study demonstrated no statistical difference between the two groups in gender and age.

Patients reacted to pain mostly by verbal expressions, or at least by frowning. Also, the investigator's interpretation of frowning and moaning might be subjective. In order to supplement this problem, the pain score was assessed by the same anesthesiologist, and we added the VAS by recalling pain 30 minutes after awakening. Nevertheless, when we checked overall incidence, only 15 of 34 patients recalled their pain, and the two groups showed no difference in pain severity. Similarly, another study reported less than $50 \%$ of patients recalled their pain, probably because of the amnesia effect of propofol. It is impossible to rule out the possibility that the pain intensity might be different if more patients recall their injection pain [10].

The results of the present study were different from that of a study on propofol-LCT injection pain [8]. Pretreatment of lidocaine with a tourniquet was more effective in preventing propofol-LCT injection pain than a premix of lidocaine. In contrast, our results show no difference in the effects of lidocaine pretreatment and premixed lidocaine on propofol-LCT/MCT injection pain. The difference between the results of the two studies may be due to the fact that propofol-LCT/MCT itself induces less pain than propofol-LCT [8]. MCT can reduce the concentration of free propofol in the aqueous phase; in a previous study, it decreased the incidence of injection pain [14]. In addition, it is already known that lidocaine can reduce propofol injection pain, although the exact mechanism is unclear. One possible mechanism is that it reversibly blocks peripheral nerve pathways at the intravenous site [15]. Furthermore, in a previous study performed with propofol-LCT, there was a difference in the incidence rate of injection pain between the pretreatment method and the premix method. This difference, however, is not clinically significant when clinical significance is defined as a difference in propofol injection pain of more than 2 points on the VAS [7]. This helps us to understand why this study had no significant difference in the severity of injection pain.

Although there have been many studies on propofol injection pain, its incidence range and preventive effects have not been constant. This is due to the differences in the methods of injection, patient characteristics, and methods of evaluation between the studies. In the present study, the incidences of pain in the pretreatment group and premix group were 13.7 and $15.4 \%$, 
respectively. The incidence and severity of injection pain in this study was similar with those of a previous study [16]. However, Krobbuaban et al. [11] reported a higher incidence of injection pain but they used different methods, for example injected 20 mg of lidocaine and did evaluations using a 4-point scale.

This study has some limitations. First, in order to reduce the probability of subjects' personal characteristics influencing the incidence of pain, the patients were not directly asked about injection pain at the very moment of injection, which may have made them unaware of the pain although they actually felt the injection pain. Second, caution should be used when applying our results to different clinical settings because of the bias between investigators evaluating injection pain even with the same pain scale. Third, in order to confirm propofol injection pain, the incidence of pain when injecting only propofol-LCT/ MCT had been studied under the same conditions as in this study. However, the purpose of this study was to compare the incidence rate of propofol-LCT/MCT injection pain between the two methods of lidocaine injection. Because of ethical considerations, propofol-LCT/MCT was not administered without lidocaine.

In conclusion, when injecting propofol-LCT/MCT, pretreating the patient with lidocaine with a tourniquet did not show an advantage in reducing injection pain compared to premixing with lidocaine.

\section{References}

1. Marik PE. Propofol: therapeutic indications and side-effects. Curr Pharm Des 2004; 10: 3639-49.

2. Picard P, Tramèr MR. Prevention of pain on injection with propofol: a quantitative systematic review. Anesth Analg 2000; 90: 963-9.

3. Fujii Y, Nakayama M. A lidocaine/metoclopramide combination decreases pain on injection of propofol. Can J Anaesth 2005; 52: 474-7.

4. Kobayashi Y, Kamada Y, Kumagai A, Seki S, Ichimiya T, Tsuchida H, et al. Pain-free injection of propofol. Masui 1998; 47: 835-8.

5. Kwak K, Kim J, Park S, Lim D, Kim S, Baek W, et al. Reduction of pain on injection of propofol: combination of pretreatment of remifentanil and premixture of lidocaine with propofol. Eur J Anaesthesiol 2007; 24: 746-50.

6. Jalota L, Kalira V, George E, Shi YY, Hornuss C, Radke O, et al. Prevention of pain on injection of propofol: systematic review and metaanalysis. BMJ 2011; 342: d1110.

7. Bachmann-Mennenga B, Ohlmer A, Boedeker RH, Mann M, Mühlenbruch B, Heesen M. Preventing pain during injection of propofol: effects of a new emulsion with lidocaine addition. Eur J Anaesthesiol 2007; 24: 33-8.

8. Allford MA, Mensah JA. Discomfort on injection: a comparison between two formulations of propofol. Eur J Anaesthesiol 2006; 23: 971-4.

9. Yew WS, Chong SY, Tan KH, Goh MH. The effects of intravenous lidocaine on pain during injection of medium- and long-chain triglyceride propofol emulsions. Anesth Analg 2005; 100: 1693-5.

10. Schaub E, Kern C, Landau R. Pain on injection: a double-blind comparison of propofol with lidocaine pretreatment versus propofol formulated with long- and medium-chain triglycerides. Anesth Analg 2004; 99: 1699-702.

11. Krobbuaban B, Siriwan D, Kumkeaw S, Tanomsat M, Jamjamrat G, Thanetses K, et al. Does addition of lidocaine to medium- and longchain triglyceride propofol emulsions significantly reduce pain on injection? J Med Assoc Thai 2008; 91: 383-7.

12. Walker BJ, Neal JM, Mulroy MF, Humsi JA, Bittner RC, McDonald SB. Lidocaine pretreatment with tourniquet versus lidocaine-propofol admixture for attenuating propofol injection pain: a randomized controlled trial. Reg Anesth Pain Med 2011; 36: 41-5.

13. Massad IM, Abu-Ali HM, Abu-Halaweh SA, Badran IZ. Venous occlusion with lidocaine for preventing propofol induced pain. A prospective double-blind randomized study. Saudi Med J 2006; 27: 997-1000.

14. Doenicke AW, Roizen MF, Rau J, O'Connor M, Kugler J, Klotz U, et al. Pharmacokinetics and pharmacodynamics of propofol in a new solvent. Anesth Analg 1997; 85: 1399-403.

15. Lai YY, Chang CL, Yeh FC. The site of action of lidocaine in intravenous regional anesthesia. Ma Zui Xue Za Zhi 1993; 31:31-4.

16. Sethi N, Jayaraman L, Sethi M, Sharma S, Sood J. Prevention of propofol pain: a comparative study. Middle East J Anesthesiol 2009; $20: 71-4$. 